\section{Triggering biofilm breakdown}

\section{By Kai-Jye Lou, Staff Writer}

Harvard University researchers have shown that a mixture of D-amino acids produced by Bacillus subtilis, a nonpathogenic bacterium commonly used in the lab, can prevent biofilm establishment and disrupt existing biofilms. ${ }^{1}$ The findings could apply to medical devices as well as to therapeutics. BASF AG has already acquired first rights for nonpharmaceutical applications.

B. subtilis can both form and disassemble biofilms, which are surfaceattached bacterial communities held together by a polymeric matrix. They can form on substrates like the inner lining of a catheter or directly on a patient's tissue.

The researchers showed that B. subtilis produces a mixture of four $\mathrm{D}$-amino acids that triggers biofilm disassembly. These $\mathrm{D}$-amino acids, which also disrupted biofilm formation in unrelated pathogenic bacterial strains, could complement existing strategies for treating and preventing infections from biofilm-associated bacteria.

Biofilm formation can be prevented with antibacterial compounds or by using engineered antifouling surfaces that kill bacteria or interfere with their ability to attach. However, dispersing established biofilms is much more challenging. In these cases, the biofilm's polymeric matrix functions as a barrier that protects the bacteria from antimicrobials and the host immune system.

Despite this protective advantage, bacteria also can trigger the disassembly of their own biofilms under specific circumstances. ${ }^{2}$ Now, a Harvard team led by Richard Losick, the Maria Moors Cabot professor of biology, thinks it has identified key molecules that trigger this process.

"Biofilms have a limited life span, and as nutrients are depleted and waste products accumulate, the bacteria in these biofilms revert to a planktonic state" in which the bacteria become unattached and free floating, said Losick. "We saw that bacteria themselves can produce factors that disperse biofilms, and we made a guess that $\mathrm{D}$-amino acids were involved in this process."

Their guess was based on findings published in Science last year by another Harvard group showing that D-amino acids modulated the synthesis of peptidoglycan, a polymer component of the bacterial cell wall. ${ }^{3}$ That group was led by Matthew Waldor, a professor of medicine at Harvard Medical School.

Losick's team treated B. subtilis cultures with D-leucine, D-methionine, D-tryptophan or D-tyrosine and found that the amino acids prevented biofilm formation at micromolar to millimolar concentrations. D-Tyrosine showed the highest potency. A mixture of the four amino acids was even more potent and had a minimum inhibitory concentration of about $10 \mathrm{nM}$.

The D-amino acids caused bacterial amyloid fibers, which link cells in the biofilm together, to disassemble.

Importantly, the D-amino acid mixture also disrupted existing B. subtilis biofilms and inhibited biofilm formation in Staphylococcus aureus and Pseudomonas aeruginosa cultures.

In contrast, a mixture of corresponding L-amino acids neither inhibited biofilm formation nor disrupted existing biofilms.

$\mathrm{D}$-Amino acids are the mirror image of L-amino acids. Amino acids produced in the body are predominantly found in the $\mathrm{L}$ isoform.

The results were published in Science.

"Many bacteria make these D-amino acids," noted Losick. "While we started in a lab strain, we showed that these amino acids also inhibit Staphylococcus and Pseudomonas biofilms. These amino acids could be a common bacterial signal for biofilm dispersal, but we don't yet know how general the effect will be."

\section{Film school}

The new work pieces together several unknowns that have muddied the understanding of bacteria biofilm biology.

"Several labs, including my own, have been studying these bacterial amyloid fibrils for years. We have known that amyloids are important for biofilm formation, but we didn't know how the bacteria triggered biofilm disassembly and reverted to planktonic existence," said Matthew Chapman, an associate professor in the Department of Molecular, Cellular and Developmental Biology at the University of Michigan. “The work from Dr. Losick's group demonstrates that D-amino acids can disrupt key interactions between amyloid fibers and the bacterial cell wall, which leads to biofilm disassembly."

"The most interesting thing in this paper to me is that specific $\mathrm{D}$-amino acids may be general dispersion signals that function in a variety of bacterial species," said E. Peter Greenberg, a professor of microbiology at the University of Washington. "Up until last fall, I didn't think about bacteria secreting these $\mathrm{D}$-amino acids into the environment. Then, all of a sudden, we learned that these extracellular molecules are being secreted, and now Losick's group is showing us something these molecules are doing."

Although the $\mathrm{D}$-amino acids showed efficacy in S. aureus and P. aeruginosa, it's unclear whether the molecules cause the bacterial amyloid fibers to disassemble, as seen with B. subtilis. "Different bacteria make their biofilms in different ways, and specific components of their biofilm matrices and the regulatory pathways involved are diverse," Losick told SciBX.

Given the heterogeneous nature of biofilms, Chapman noted that "currently, there is no reason to assume that the D-amino acid-mediated biofilm disassembly mechanism described for Bacillus is the same one responsible for biofilm disassembly in Staphylococcus or Pseudomonas."

Waldor agreed that a universal mechanism of biofilm dispersal is unlikely. Thus, he said the effect of D-amino acids needs to be directly studied in a variety of $P$. aeruginosa and $S$. aureus strains, as well as in other unrelated bacteria. 


\section{ANALYSIS}

From a clinical standpoint, Waldor said it will be important to see how well the D-amino acids prevent biofilm formation in medical catheters and in animal models of biofilm infection. "One can imagine making catheters that contain or release $\mathrm{D}$-amino acids to prevent the formation of biofilms," he said.

Losick said the route of delivery would likely vary with the indication. "For example, you could design a catheter that releases D-amino acids. For something like bacterial endocarditis, I would imagine giving the mixture systemically. For Pseudomonas infections in the lung, I can imagine giving the mixture as an inhalant," he said.

\section{The road to applications}

Although more studies in pathogenic bacteria are needed to establish the universality of the approach, D-amino acids already possess several properties that make them attractive development candidates.

Chapman noted that $\mathrm{D}$-amino acids are naturally produced by almost all cell types. He added that the mixture of D-amino acids used in the study was resistant to heat and other potential denaturants, suggesting that $\mathrm{D}$-amino acids would be suitably stable in diverse environments.

Moreover, Chapman thinks that because the D-amino acids did not appear to affect bacterial growth, they likely will exert less selective pressure to develop resistance than antibiotics. "If bacteria are placed under a biofilm-selective pressure, they may be less likely to develop resistance than if they were placed under a life-or-death situation," said Chapman.

"We have been unable to produce mutants that are resistant to a mixture containing all four D-amino acids," added Losick. "This is a good indicator that bacteria could have difficultly developing resistance against these amino acids."

Both Chapman and Losick think D-amino acids are likely to have a good safety profile. For example, Losick noted that certain food products in Asia are manufactured using a commercial strain of B. subtilis and contain D-amino acids.
A case in point is natto, a Japanese dish consisting of fermented soybeans. "This dish is regularly eaten and contains $\mathrm{D}$-amino acids, so I suspect that a mixture of $\mathrm{D}$-amino acids would be safe for use in humans," Losick said.

However, Chapman acknowledged that "nobody knows enough about the effects from ingesting an amount of $\mathrm{D}$-amino acids needed to have an antibiofilm effect. We also don't know how toxic these D-amino acids will be to other cell types."

Losick said his group is now trying to identify the factors that control the timing of $\mathrm{D}$-amino acid production in bacteria and is studying the mechanism of action of the D-amino acids in other bacterial strains, including S. aureus and P. aeruginosa.

Greenberg said an important next step would be to evaluate the effect of the D-amino acids on biofilms grown under flow conditions. "Biofilms in humans form under conditions where fluid flows over them. This flow actually makes the biofilm stick better and also brings nutrients to the bacteria," he said.

Harvard has filed a patent application covering the use of D-amino acids to prevent and disperse biofilms. The work is available for licensing.

Lou, K.-J. SciBX 3(19); doi:10.1038/scibx.2010.576

Published online May 13, 2010

\section{REFERENCES}

1. Kolodkin-Gal, I. et al. Science; published online April 29, 2010; doi:10.1126/science.1188628

Contact: Richard Losick, Harvard University, Cambridge, Mass. e-mail: losick@mcb.harvard.edu

2. Karatan, E. \& Watnick, P. Microbiol. Mol. Biol. Rev. 73, 310-347 (2009)

3. Lam, H. et al. Science 325,1552-1555 (2009)

COMPANIES AND INSTITUTIONS MENTIONED

BASF AG (NYSE:BF), Ludwigshafen, Germany

Harvard Medical School, Boston, Mass.

Harvard University, Cambridge, Mass.

University of Michigan, Ann Arbor, Mich.

University of Washington, Seattle, Wash. 